\title{
INFERRED SURVIVAL ANALYSIS OF METASTATIC BREAST CANCER IN A BRAZILIAN EXTERNAL LIBRARY
}

\author{
ANÁLISIS DE SOBREVIDA INFERIDA DE CÂNCER DE MAMA METASTÁTICO EN UNA \\ BIBLIOTECA EXTERNA BRASILEÑA
}

Marcio Debiasi ${ }^{1}$, Sergio Simon ${ }^{2}$, Luciana Preger ${ }^{3}$, Lourenia Cassoli ${ }^{4}$ Carlos Barrios ${ }^{5}$

\begin{abstract}
Objective: To infer overall survival (OS) and progression free survival (PFS) of patients with metastatic breast cancer who have started first line treatment between January 2009 and July 2010 in the Brazilian public healthcare system (SUS). Methods: This is a non-interventional, retrospective study, developed based on DATASUS, a Brazilian public claims database, that gathers information from all hospitals and outpatient providers reimbursed by SUS. Patients with breast cancer diagnosis $(I C D 10=C 50)$ starting first line treatment for metastatic disease between January 2009 and July 2010 were included in the analysis, so that they would have a relatively uniform follow-up of five years in the database. OS was defined as the time from the start of first line therapy for metastatic disease until the last treatment received for breast cancer or the last patient record in the database, irrespective of the type of intervention or diagnosis. PFS was defined as the time from the start of first line therapy for metastatic disease until the change of treatment regimen (change of drug used or inclusion of a new drug to the previously used regimen). Survival curves were built using the Kaplan-Meier method. Results: 15,696 patients were included in the analysis (average age $=55$ years, 99\% women). The median OS was 15 months and patients used on average 1.44 treatment lines until loss of follow-up. Median PFS was 4 months. Among patients included in the analysis, $94.2 \%$ started first line treatment with chemotherapy and 5.8\% with hormone therapy. Conclusion: OS and PFS inferred for patients with metastatic breast cancer starting first line treatment in the Brazilian SUS was lower than international references, which may be related to the lack of access to the most efficacious therapies for this disease stage, especially for HER2 positive patients.
\end{abstract}

Keywords: Breast neoplasms; Survival; Disease-free survival; Administrative claims; Healthcare

\footnotetext{
1. LACOG (Latin American Cooperative Oncology Group) e Escola de Medicina da PUCRS

2. Hospital Israelita Albert Einstein e Centro Paulista de Oncologia

3. Ex-funcionária da Roche, São Paulo, Brasil

4. Roche, São Paulo, Brasil

5. FAMED PUCRS. Hospital do Câncer Mãe de Deus, PORTO ALEGRE, RS Brasil
}

Financial support: Este estudo foi financiado por Produtos Roche Químicos e Farmacêuticos S.A.

Conflicts of interest: The authors declare no conflict of interest relevant to this manuscript.

Correspondence author: Marcio Debiasi. Av Ipiranga, 6681 - prédio 99A / Sala 806 - CEP 90619-900 - Porto Alegre. FONE: (51) 3384-5334 / FAX (51) 3384 1855. marciomed1@hotmail.com 


\section{RESUMO}

Objetivo: inferir a sobrevida global (SG) e a sobrevida livre de progressão (SLP) de pacientes com câncer de mama metastático que iniciaram o tratamento de primeira linha entre janeiro de 2009 e julho de 2010 no sistema único de saúde brasileiro (SUS). Métodos: Estudo retrospectivo não intervencionista, desenvolvido com base no banco de dados do DATASUS, uma base de dados pública para fins de reembolso que reúne informações de todos os estabelecimentos públicos do país. Foram incluídos pacientes com diagnóstico de câncer de mama $(C I D 10=C 50)$ iniciando o tratamento de primeira linha para doença metastática entre janeiro de 2009 e julho de 2010. A SG foi definida como o tempo desde o início da terapia de primeira linha para doença metastática até o último tratamento recebido para câncer de mama ou o último registro de paciente no banco de dados, independentemente do tipo de intervenção ou diagnóstico. A SLP foi definida como o tempo desde o início da terapia de primeira linha para a doença metastática até a mudança do regime de tratamento (alteração do medicamento usado ou inclusão de um novo fármaco para o regime anteriormente utilizado). As curvas de sobrevida foram construídas através do método de Kaplan-Meier. Resultados: 15.696 pacientes foram incluídos na análise (idade média = 55 anos, 99\% mulheres). A SG média foi de 15 meses e os pacientes usaram em média 1,44 linha de tratamento até a perda de seguimento. A SLP média foi de 4 meses. Entre os pacientes incluídos na análise, 94,2\% iniciaram o tratamento de primeira linha com quimioterapia e 5,8\% com terapia hormonal. Conclusão: A SG e SLP inferidas para pacientes com câncer de mama metastático iniciando o tratamento de primeira linha no SUS foi inferior às referências internacionais, o que pode estar relacionado à falta de acesso às terapias mais eficazes para este estágio da doença, especialmente para pacientes HER2+.

Descritores: Neoplasias da mama; Sobrevida; Sobrevida livre de doença; Processos administrativos; Saúde

\section{INTRODUÇÃO}

O câncer de mama é a neoplasia mais incidente e responsável pelo maior número de mortes por câncer entre mulheres globalmente.1,2 No Brasil, de acordo com o Instituto Nacional do Câncer (INCA), excluindo os tumores de pele não melanoma, o câncer de mama é a neoplasia mais comum no sexo feminino, com uma incidência de 68,2 casos a cada 100 mil mulheres em 2016. ${ }^{1}$ A taxa de mortalidade por câncer de mama no Brasil é elevada, com cerca de 16,3 óbitos por 100.000 mulheres em 2013, o que pode estar relacionado ao grande número de diagnósticos em fases avançadas da doença. 3,4

O estadiamento do câncer de mama leva em conta o tamanho do tumor, o comprometimento de linfonodos e a existência de metástases à distância, podendo ser classificado de forma simplificada como Estadio 0 (carcinoma ductal in situ), Estadio 1 (tumor $<2 \mathrm{~cm}$, com linfonodos axilares negativos), Estadio 2, (tumor $<2 \mathrm{~cm}$ com linfonodos axilares positivos, ou tumor de 2-5 cm com axila positiva ou negativa, ou tumor $>5$ $\mathrm{cm}$ com axila negativa), Estadio 3 (tumor $>5 \mathrm{~cm}$ e envolvimento dos gânglios linfáticos axilares ipsilaterais) e Estadio 4 (metástases à distância). ${ }^{5} \mathrm{O}$ estadiamento, além de auxiliar na determinação do prognóstico e no planejamento do tratamento, uniformiza grupos de pacientes, facilitando estudos comparativos entre os mesmos. ${ }^{5}$ Globalmente, cerca de 6 a $10 \%$ dos pacientes diagnosticados com câncer de mama já se apresentam com doença metastática (estadio IV). Este subgrupo tem uma baixa probabilidade de sobrevida em 5 anos, da ordem de 20\%. ${ }^{2}$ A progressão das metástases à distância representa a principal causa de morte neste grupo. ${ }^{6}$ Além disso, de 25 a 30\% dos pacientes tratados em estadios mais iniciais da doença desenvolvem recorrência sistêmica. ${ }^{7}$

No Brasil, estima-se que a proporção de pacientes com doença metastática seja ainda maior do que nos países desenvolvidos, de onde se originam a maior parte das estatísticas. Isto provavelmente se dá em virtude dos diagnósticos tardios e da restrição de acesso aos tratamentos adjuvantes em tempo adequado. ${ }^{3} \mathrm{~A}$ proporção de pacientes com câncer de mama avançado é maior no setor público de saúde quando comparado ao setor privado. Em centros públicos, 13,8\% das pacientes se apresentam em estadio I, enquanto que em centros privados esta proporção sobe para 34,1\%. Em contrapartida, estadios III e IV são mais frequen- 
tes nos centros públicos do que nos privados (estadio III: 30,9\% versus 12,7\%; estadio IV: $6,0 \%$ versus $3,5 \%){ }^{8}$

Tomando-se em consideração o acima exposto, conclui-se que existe um grande contingente de mulheres com câncer de mama metastático que necessitam de tratamento no Brasil. Neste cenário, o tratamento tem como objetivos prolongar a sobrevida e melhorar a qualidade de vida, proporcionando às pacientes o maior tempo possível sem os sintomas da doença. ${ }^{9}$ A abordagem atual consiste em adequar o tratamento de acordo com as características particulares de cada paciente. ${ }^{10}$ Tal como no câncer de mama inicial, as características imunohistoquímicas do tumor devem ser tomadas em consideração na tomada de decisão em relação tratamento proposto: quimioterapia, hormonioterapia ou terapia anti-HER2, isolados ou em combinação. ${ }^{10}$ Os principais fatores a serem avaliados são a expressão de HER-2 e de receptores hormonais, os locais de metástases (predominantemente visceral versus óssea/tecidos moles), o estado de menopausa, os tipos de tratamentos administrados anteriormente, a tolerância aos tratamentos anteriores, a existência de comorbidades e o intervalo livre de doença. ${ }^{10}$ No Sistema de Saúde Pública no Brasil, considerando o perfil imunohistoquímico do tumor para definição do tratamento, exista uma necessidade médica não atendida para as pacientes com doença metastática HER2 positiva, visto que terapias alvo anti-HER2 não eram disponibilizadas para pacientes com doença metastática. Felizmente, esta situação mudou com a publicação da portaria N 29 de 02 de agosto de 2017, que incorpora o trastuzumabe para o tratamento do câncer de mama metastático em primeira linha.

Tendo em vista que informações sobre desfechos clínicos alcançados por pacientes com câncer de mama metastático tratados no SUS são limitadas, este estudo foi desenvolvido com o objetivo de inferir a sobrevida global (SG) e a sobrevida livre de progressão (SLP) de pacientes com câncer de mama metastático que tenham iniciado o tratamento de primeira linha entre janeiro de 2009 e julho de 2010 pelo Sistema Único de Saúde (SUS) utilizando-se a base de dados do SUS (DATASUS).

\section{MÉTODOS}

Este é um estudo não intervencional, retrospectivo, em bases de dados secundárias, para avaliar o cenário de primeira linha de tratamento do câncer de mama metastático no SUS utilizando o DATASUS. O DATASUS é um conjunto de banco de dados público que recebe informações de todos os hospitais brasileiros e prestadores ambulatoriais que são reembolsados pelo sistema público de saúde. Qualquer internação, procedimento de alta complexidade, tratamento de alto custo em oncologia que seja realizado no ambiente público deve ser registrado através deste sistema para a solicitação de reembolso. O pedido na configuração ambulatorial é denominado APAC - Autorização para o Procedimento de Alta Complexidade. O DATASUS é alimentado com todas essas solicitações e cada pedido é ligado a um paciente específico. Apesar da quantidade significativa de informação no DATASUS, não há dado específico disponível sobre o estado imunohistoquímico de cada paciente e a ausência de dados sobre a evolução clínica do paciente na base faz com que seja difícil a localização de relatos de eventos adversos e a definição de desfechos.

Foram considerados elegíveis para inclusão na análise os pacientes com diagnóstico de câncer de mama (CID 10: C50) cuja primeira linha de tratamento sistêmico para doença metastática foi iniciada entre janeiro de 2009 e julho de 2010. Foram consideradas em "primeira linha de tratamento paliativo" aquelas para as quais não havia registro de tratamentos prévios para doença metastática na base de dados por pelo menos um ano. Definiu-se o período de inclusão entre janeiro de 2009 e julho de 2010 a fim de garantir que as pacientes tivessem um tempo de seguimento relativamente uniforme de cinco anos na base de dados. A data de corte para esta análise foi fevereiro de 2016. Assim, os pacientes foram seguidos até que não houvesse mais registros de tratamentos ou por cinco anos na base de dados.

A presente análise tem como objetivos co-primários sobrevida global (SG) e sobrevida livre de progressão (SLP). Em virtude das limitações decorrentes do uso da base de dados, SG foi definida como o tempo entre o primeiro tratamento em primeira linha para a doença metastática e o final do seguimento ou até a data do último registro localizado para o paciente na base de dados, independente da natureza da intervenção. Nos casos em que o último tratamento documentado para o paciente ocorresse antes do término do seguimento, foram pesquisados outros procedimentos realizados em ambiente ambulatorial, independente do diagnóstico, como evidência de que o paciente permanecia vivo. Quando não eram identificados novos procedimentos, o paciente era dado como óbito. Já a SLP foi definida como o tempo entre o início do tratamento de primeira linha para a doença metastática e a data da troca do regime de tratamento para o início da segunda linha. Considerou-se "trocado o regime de tratamento" quando houvesse a troca do regime utilizado ou a adição de um novo medicamento à terapia previamente utilizada. As curvas de sobrevida foram construídas pelo método de Kaplan-Meier.

Foram excluídos pacientes iniciando tratamento para doença metastática em segunda linha. As características dos pacientes incluídos na análise foram descritas em relação a gênero e faixa etária.

Dado o caráter retrospectivo do estudo, que foi baseado em revisão de dados publicamente disponíveis, não houve necessidade de avaliação por Comitê de Ética em Pesquisa nem aplicação de termo de consentimento livre e esclarecido, de acordo com a resolução no 510 de 7 de abril de 2016.11 


\section{RESULTADOS}

Entre janeiro de 2009 e julho de 2010, 15.696 pacientes iniciaram primeira linha de tratamento sistêmico para câncer de mama avançado. O processo de seleção dos pacientes para a obtenção da coorte efetivamente analisada é apresentado na Figura 1. As distribuições por idade e por gênero são descritas na Figura 2. A idade média ( $\pm D P$ ) dos pacientes foi de 55 $( \pm 13)$ anos e $99 \%$ eram do sexo feminino.
A mediana de SG foi de 15 meses para os 15.696 pacientes incluídos na análise (Figura 3). As pacientes passaram, em média, por 1,44 linhas de tratamento (mediana = 1 linha de tratamento; máximo = $5 \mathrm{li}$ nhas) até a perda de seguimento. A mediana de SLP foi de 4 meses para a mesma população (Figura 4).

Entre os pacientes incluídos na análise, 94,2\% iniciaram tratamento de primeira linha metastática com quimioterapia e se mantiveram em tratamento no

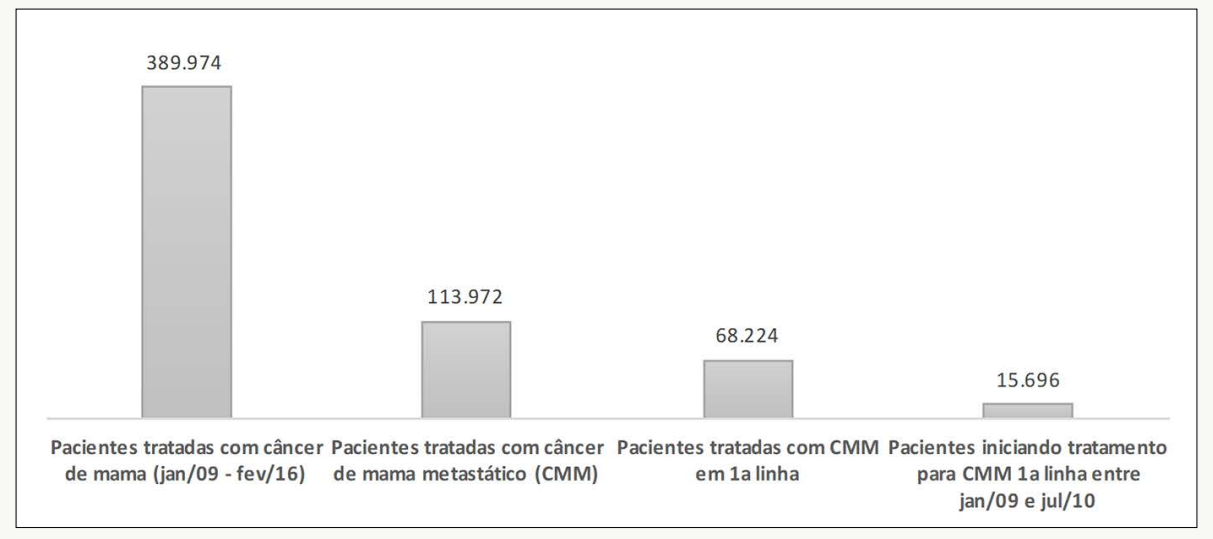

Figura 1. Processo de seleção da coorte de pacientes para a análise.

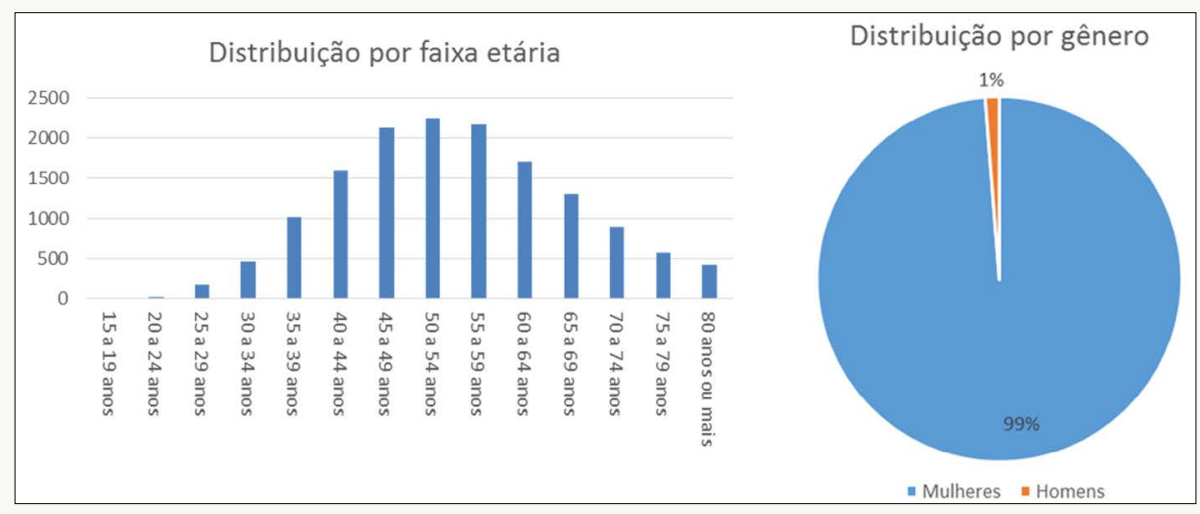

Figura 2. Distribuição por faixa etária e gênero de pacientes iniciando a primeira linha de tratamento para o câncer de mama metastático. DATASUS, janeiro/09 a julho/10.

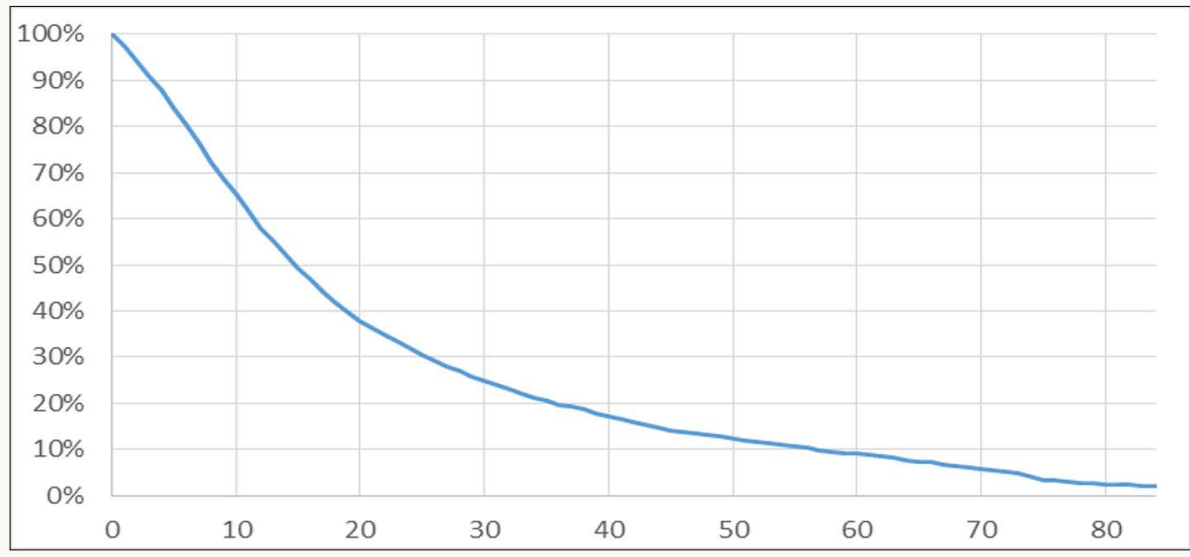

Figura 3. Sobrevida global. DATASUS, janeiro 2009 a fevereiro 2016. 


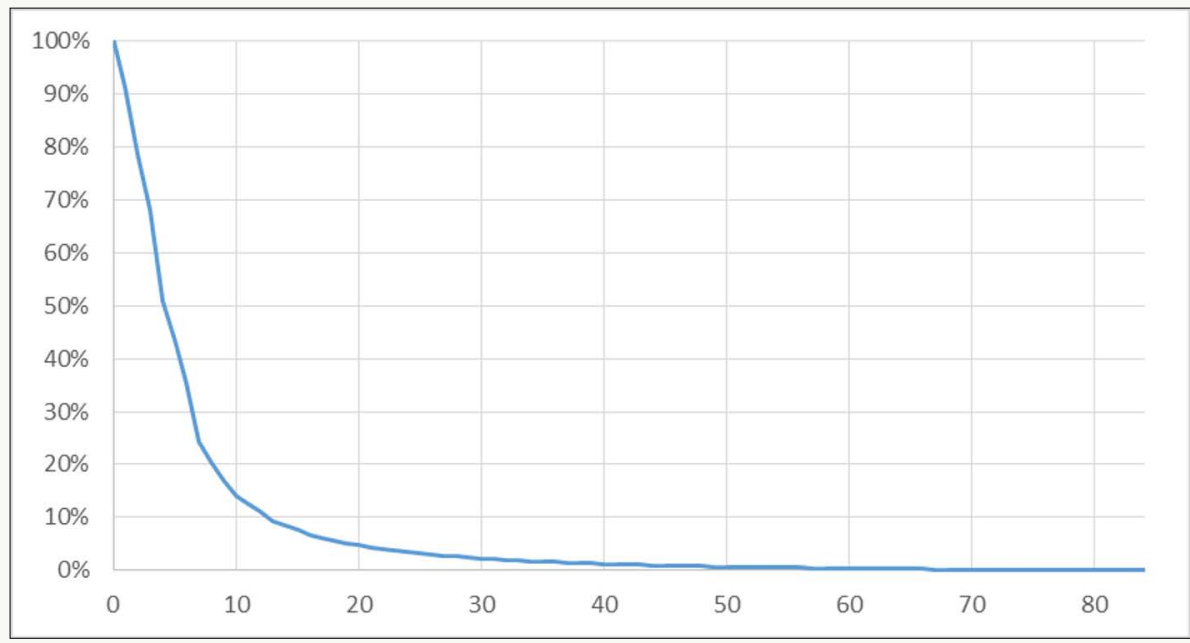

Figura 4. Sobrevida livre de progressão. DATASUS, janeiro 2009 a fevereiro 2016.

mesmo regime por uma mediana de quatro meses. Destes, $28 \%$ alteraram para hormonioterapia, permanecendo em tratamento por uma mediana de nove meses.

Os 5,8\% restantes, identificados no banco de dados, iniciaram hormonioterapia de primeira linha e permaneceram em tratamento com o mesmo regime por um tempo mediano de quatro meses. Destes, $79 \%$ foram tratados com quimioterapia posteriormente, permanecendo em tratamento por uma mediana de sete meses, e o restante (21\%) migrou para hormonioterapia de segunda linha, permanecendo em tratamento por uma mediana de cinco meses. Menos de $1 \%$ dos pacientes iniciaram tratamento concomitante com hormonioterapia e quimioterapia e posteriormente passaram a receber somente um dos dois tratamentos.

Foi realizada uma análise, de forma agrupada, para identificar os tratamentos mais utilizados (>10\%) em primeira linha. Taxanos (24\%), capecitabina (22\%) e fluorouracil + doxorrubicina + ciclofosfamida (12\%) foram os mais frequentemente identificados. Em segunda linha, os mais frequentes foram capecitabina (20\%), taxanos (17\%) e tamoxifeno (15\%).

\section{DISCUSSÃO}

Nas últimas décadas, foi observada uma importante evolução nas opções de tratamento para o câncer de mama metastático, em especial para pacientes com câncer de mama HER2 positivo. Em virtude da escassez de dados locais referentes aos desfechos dos pacientes tratados com câncer de mama avançado no SUS, o presente estudo teve por objetivo estimar a SG e a SLP de pacientes a partir dos dados disponíveis no DATASUS e tentar traçar um paralelo entre esses dados e a literatura internacional acerca do tema. Neste cenário, a mediana de SLP e de SG nesta coorte foi 4 e 15 meses, respectivamente. Posto que o SUS não oferecia terapias-alvo para estes pacientes à época em que as pacientes aqui analisadas foram tratadas e que as informações referentes ao perfil imunohistoquímico dos tumores não são disponíveis na base de dados pesquisada, esses resultados serão primeiramente colocados em paralelo com estudos internacionais descrevendo desfechos de pacientes antes do advento destas terapias.

Andre e cols 12 reportaram SG mediana de 23 meses quando avaliaram 343 mulheres com câncer de mama metastático de novo diagnosticadas entre 1987 e 1993 e de 29 meses para 381 mulheres diagnosticadas entre 1994 e 2000. Já o estudo publicado em 1991 por Muss e cols,,$^{13}$ portanto também anterior às recomendações de utilização de terapias alvo, mostrou SLP de 9,4 meses para pacientes com câncer de mama metastático.

Uma metanálise publicada em 2015 por Adunlin $e$ cols ${ }^{14}$ avaliou os desfechos de SG e SLP para diversos estudos que utilizaram antraciclinas, taxanos ou terapias alvo para tratamento do câncer de mama. Entre os estudos incluídos na metanálise, foram selecionados aqueles realizados em populações não selecionadas por status de receptor hormonal (HR) ou de expressão do HER2, em primeira linha, e seus respectivos braços de tratamento que se encaixavam entre os tipos de tratamento mais prevalentes no SUS, como anteriormente detalhado. Estes estão apresentados na Tabela 1.

Como pode ser observado na Tabela 1, os estudos que avaliaram a SG e SLP de pacientes com câncer de mama metastático tratados em primeira linha apresentaram resultados de SLP mediana que variaram de 3,4 meses a 14,3 meses, com uma média de 7,8 meses. Já a SG mediana variou de 11,8 meses a 41 meses com uma média de 20,8 meses. 
Tabela 1. Estudos de referência que avaliaram a SG e SLP de pacientes com câncer de mama metastático tratados em primeira linha.

\begin{tabular}{|c|c|c|c|c|c|c|}
\hline AUTOR & ANO & TRATAMENTO & POPULAÇÃO & $\mathrm{N}$ & SG mediana & SLP mediana \\
\hline Blomqvist & 1993 & $\begin{array}{c}\mathrm{D}=\text { fluorouracil } 500 \mathrm{mg} / \mathrm{m} 2 \mathrm{q} 4 \mathrm{w}+\text { epiru- } \\
\text { bicin } 60 \mathrm{mg} / \mathrm{m} 2 \mathrm{q} 4 \mathrm{w}+\text { cyclophosphamide } \\
500 \mathrm{mg} / \mathrm{m} 2 \mathrm{q} 4 \mathrm{w}\end{array}$ & $\mathrm{HR} \pm$ / HER2 NI & 84 & 21,2 & 5,4 \\
\hline Blomqvist & 1993 & $\begin{array}{c}\mathrm{C}=\text { fluorouracil } 500 \mathrm{mg} / \mathrm{m} 2 \mathrm{q} 1 \mathrm{w}+\text { epiru- } \\
\text { bicin } 60 \mathrm{mg} / \mathrm{m} 2 \mathrm{q} 1 \mathrm{w}+\text { cyclophosphamide } \\
500 \mathrm{mg} / \mathrm{m} 2 \mathrm{q} 1 \mathrm{w}\end{array}$ & HR \pm / HER2 NI & 86 & 11,8 & 9,2 \\
\hline Brufman & 1997 & $\begin{array}{c}\mathrm{D} \text { = fluorouracil } 500 \mathrm{mg} / \mathrm{m} 2 \mathrm{q} 3 \mathrm{w}+\text { epirubi- } \\
\text { cin } 100 \mathrm{mg} / \mathrm{m} 2 \mathrm{q} 3 \mathrm{w}+\text { cyclophosphamide } \\
500 \mathrm{mg} / \mathrm{m} 2 \mathrm{q} 3 \mathrm{w}\end{array}$ & HR \pm / HER2 NI & 212 & 18 & 5,8 \\
\hline Brufman & 1997 & $\begin{array}{c}\mathrm{C}=\text { fluorouracil } 500 \mathrm{mg} / \mathrm{m} 2 \mathrm{q} 3 \mathrm{w}+\text { epiru- } \\
\text { bicin } 50 \mathrm{mg} / \mathrm{m} 2 \mathrm{q} 3 \mathrm{w}+\text { cyclophosphamide } \\
500 \mathrm{mg} / \mathrm{m} 2 \mathrm{q} 3 \mathrm{w}\end{array}$ & HR \pm / HER2 NI & 242 & 17 & 5,3 \\
\hline Bishop & 1999 & $\mathrm{D}=$ paclitaxel $200 \mathrm{mg} / \mathrm{m} 2 \mathrm{IV}$ q3w & $\mathrm{HR} \pm$ / HER2 NI & 107 & 17,3 & 5,3 \\
\hline Blajman & 1999 & $\begin{array}{c}\mathrm{D}=\text { fluorouracil } 500 \mathrm{mg} / \mathrm{m} 2 \mathrm{IV}+\text { doxorubi- } \\
\text { cin } 50 \mathrm{mg} / \mathrm{m} 2 \mathrm{IV}+\text { cyclophosphamide } 500 \\
\text { mg/m2 IV }\end{array}$ & HR \pm / HER2 NI & 85 & 17,8 & 7,5 \\
\hline Smith & 1999 & $\mathrm{D}=$ paclitaxel $250 \mathrm{mg} / \mathrm{m} 2 \mathrm{q} 3 \mathrm{w}$ & $\mathrm{NI}$ & 284 & 21,9 & 7,2 \\
\hline Smith & 1999 & $\mathrm{C}=$ paclitaxel $250 \mathrm{mg} / \mathrm{m} 2 \mathrm{q} 3 \mathrm{w}$ & $\mathrm{NI}$ & 279 & 21,1 & 6,3 \\
\hline French Epi Gp & 2000 & $\begin{array}{c}\mathrm{D} \text { = fluorouracil } 500 \mathrm{mg} / \mathrm{m} 2 \mathrm{q} 3 \mathrm{w}+\text { epirubi- } \\
\text { cin } 100 \mathrm{mg} / \mathrm{m} 2 \mathrm{q} 3 \mathrm{w}+\text { cyclophosphamide } \\
500 \mathrm{mg} / \mathrm{m} 2 \mathrm{q} 3 \mathrm{w}\end{array}$ & $\mathrm{NI}$ & 145 & 18,9 & 8,3 \\
\hline French Epi Gp & 2000 & $\begin{array}{c}\mathrm{C}=\text { fluorouracil } 500 \mathrm{mg} / \mathrm{m} 2 \mathrm{q} 3 \mathrm{w}+\text { epiru- } \\
\text { bicin } 75 \mathrm{mg} / \mathrm{m} 2 \mathrm{q} 3 \mathrm{w}+\text { cyclophosphamide } \\
500 \mathrm{mg} / \mathrm{m} 2 \mathrm{q} 3 \mathrm{w}\end{array}$ & $\mathrm{NI}$ & 139 & 17,9 & 10,3 \\
\hline French Epi Gp & 2000 & $\begin{array}{c}\mathrm{D} \text { = fluorouracil } 500 \mathrm{mg} / \mathrm{m} 2 \mathrm{q} 3 \mathrm{w}+\text { epirubi- } \\
\text { cin } 100 \mathrm{mg} / \mathrm{m} 2 \mathrm{q} 3 \mathrm{w}+\text { cyclophosphamide } \\
500 \mathrm{mg} / \mathrm{m} 2 \mathrm{q} 3 \mathrm{w}\end{array}$ & $\mathrm{NI}$ & 133 & 16,3 & 6,2 \\
\hline French Epi Gp & 2000 & $\begin{array}{c}\mathrm{C}=\text { fluorouracil } 500 \mathrm{mg} / \mathrm{m} 2 \mathrm{q} 3 \mathrm{w}+\text { epiru- } \\
\text { bicin } 75 \mathrm{mg} / \mathrm{m} 2 \mathrm{q} 3 \mathrm{w}+\text { cyclophosphamide } \\
500 \mathrm{mg} / \mathrm{m} 2 \mathrm{q} 3 \mathrm{w}\end{array}$ & $\mathrm{NI}$ & 139 & 17,9 & 10,3 \\
\hline Paridaens & 2000 & $\mathrm{D}=$ Paclitaxel $200 \mathrm{mg} / \mathrm{m} 2 \mathrm{IV}$ q3w & $\mathrm{HR} \pm$ / HER2 NI & 166 & 18,3 & 7,5 \\
\hline Ackland & 2001 & $\begin{array}{l}\text { D = fluorouracil } 500 \text { mg/m2 IV + epirubicin } 50 \\
\text { mg/m2 IV + cyclophosphamide } 400 \text { mg/m2 IV }\end{array}$ & HR \pm / HER2 NI & 223 & 20,1 & 8,7 \\
\hline Batist & 2001 & $\begin{array}{c}\mathrm{C}=\text { doxorubicin } \mathrm{q} 3 \mathrm{w}+\text { cyclophosphamide } \\
\text { of } 600 \mathrm{mg} / \mathrm{m} 2 \mathrm{q} 3 \mathrm{w}\end{array}$ & HR \pm / HER2 NI & 155 & 16 & 5,5 \\
\hline Del Mastro & 2001 & $\begin{array}{c}\mathrm{D}=\text { fluorouracil } 600 \mathrm{mg} / \mathrm{m} 2 \mathrm{q} 2 \mathrm{ws}+\text { epiru- } \\
\text { bicin } 80 \mathrm{mg} / \mathrm{m} 2 \mathrm{q} 2 \mathrm{w}+\text { cyclophosphamide } \\
1000 \mathrm{mg} / \mathrm{m} 2 \mathrm{q} 2 \mathrm{w}\end{array}$ & $\mathrm{NI}$ & 77 & 27,2 & 12,8 \\
\hline Del Mastro & 2001 & $\begin{array}{c}\mathrm{C}=\text { fluorouracil } 600 \mathrm{mg} / \mathrm{m} 2 \mathrm{q} 3 \mathrm{w}+\text { epiru- } \\
\text { bicin } 60 \mathrm{mg} / \mathrm{m} 2 \mathrm{q} 3 \mathrm{w}+\text { cyclophosphamide } \\
600 \mathrm{mg} / \mathrm{m} 2 \mathrm{q} 3 \mathrm{w}\end{array}$ & $\mathrm{NI}$ & 74 & 32,7 & 14,3 \\
\hline Fountzilas & 2001 & $\begin{array}{c}\mathrm{D}=\text { epirubicin } 110 \mathrm{mg} / \mathrm{m} 2 \mathrm{q} 2 \mathrm{w}+\text { paclitaxel } \\
225 \mathrm{mg} / \mathrm{m} 2 \mathrm{q} 2 \mathrm{w}\end{array}$ & HR \pm / HER2 NI & 93 & 21,5 & 10 \\
\hline Fountzilas & 2001 & $\begin{array}{c}\mathrm{C}=\text { epirubicin } 80 \mathrm{mg} / \mathrm{m} 2 \mathrm{q} 3 \mathrm{w}+\text { paclitaxel } \\
175 \mathrm{mg} / \mathrm{m} 2\end{array}$ & HR \pm / HER2 NI & 90 & 20 & 8,5 \\
\hline Jassem & 2001 & $\begin{array}{c}\mathrm{D}=\text { doxorubicin } 50 \mathrm{mg} / \mathrm{m} 2 \mathrm{q} 3 \mathrm{w}+\text { paclitaxel } \\
220 \mathrm{mg} / \mathrm{m} 2 \mathrm{q} 3 \mathrm{w}\end{array}$ & $\mathrm{NI}$ & 134 & 23 & 8,1 \\
\hline Jassem & 2001 & $\begin{array}{c}\mathrm{C}=\text { fluorouracil } 500 \mathrm{mg} / \mathrm{m} 2 \mathrm{q} 3 \mathrm{w}+\text { doxoru- } \\
\text { bicin } 50 \mathrm{mg} / \mathrm{m} 2 \mathrm{q} 3 \mathrm{w}+\text { cyclophosphamide } \\
500 \mathrm{mg} / \mathrm{m} 2 \mathrm{q} 3 \mathrm{w}\end{array}$ & $\mathrm{NI}$ & 133 & 18,3 & 6,2 \\
\hline Namer & 2001 & $\begin{array}{c}\mathrm{C}=\text { fluorouracil } 500 \mathrm{mg} / \mathrm{m} 2 \mathrm{IV}+\text { doxorubicin } \\
\text { or epirubicin } 50 \mathrm{mg} / \mathrm{m} 2 \mathrm{IV}+\text { cyclophospha- } \\
\text { mide } 500 \mathrm{mg} / \mathrm{m} 2 \mathrm{IV}\end{array}$ & $\mathrm{NI}$ & 139 & 20 & 7 \\
\hline
\end{tabular}


continuação...

Tabela 1. Estudos de referência que avaliaram a SG e SLP de pacientes com câncer de mama metastático tratados em primeira linha.

\begin{tabular}{|c|c|c|c|c|c|c|}
\hline AUTOR & ANO & TRATAMENTO & POPULAÇÃO & $\mathrm{N}$ & SG mediana & SLP mediana \\
\hline Biganzoli & 2002 & $\begin{array}{c}\mathrm{D}=\text { doxorubicin } 60 \mathrm{mg} / \mathrm{m} 2 \mathrm{IV} \mathrm{q} 3 \mathrm{w}+\text { pacli- } \\
\text { taxel } 175 \mathrm{mg} / \mathrm{m} 2 \mathrm{q} 3 \mathrm{w}\end{array}$ & $\mathrm{HR} \pm$ / HER2 NI & 138 & 20,6 & 6 \\
\hline Biganzoli & 2002 & $\begin{array}{c}\mathrm{C}=\text { doxorubicin } 60 \mathrm{mg} / \mathrm{m} 2 \mathrm{IV} \text { q3w }+ \text { cyclo- } \\
\text { phosphamide } 600 \mathrm{mg} / \mathrm{m} 2 \mathrm{q} 3 \mathrm{w}\end{array}$ & HR \pm / HER2 NI & 137 & 20,5 & 6 \\
\hline Nabholtz & 2003 & $\begin{aligned} \mathrm{D}= & \text { doxorubicin } 50 \mathrm{mg} / \mathrm{m} 2 \mathrm{q} 3 \mathrm{w}+ \\
& \text { docetaxel } 75 \mathrm{mg} / \mathrm{m} 2 \mathrm{q} 3 \mathrm{w}\end{aligned}$ & $\mathrm{NI}$ & 214 & 22,5 & 8,7 \\
\hline Nabholtz & 2003 & $\begin{array}{c}\mathrm{C}=\text { doxorubicin } 60 \mathrm{mg} / \mathrm{m} 2+\text { cyclophospha- } \\
\text { mide } 600 \mathrm{mg} / \mathrm{m} 2\end{array}$ & $\mathrm{NI}$ & 215 & 21,7 & 7,44 \\
\hline Parnes & 2003 & $\begin{array}{c}\text { D = fluorouracil } 200 \text { mg/m2 IV + doxoru- } \\
\text { bicin } 40 \text { mg/m2 + cyclophosphamide } 500 \\
\text { mg/m2 + leucovorin } 200 \text { mg/m2 in } 100 \text { mL } \\
\text { IV }\end{array}$ & HR \pm / HER2 NI & 121 & 20,52 & 9,48 \\
\hline Parnes & 2003 & $\begin{array}{c}\mathrm{C}=\text { fluorouracil } 200 \mathrm{mg} / \mathrm{m} 2 \mathrm{IV}+\text { doxoru- } \\
\text { bicin } 40 \mathrm{mg} / \mathrm{m} 2+\text { cyclophosphamide } 500 \\
\mathrm{mg} / \mathrm{m} 2\end{array}$ & HR \pm / HER2 NI & 121 & 20,64 & 9 \\
\hline Chan & 2004 & $\begin{array}{c}\mathrm{C}=\text { epirubicin } 75 \mathrm{mg} / \mathrm{m} 2+\text { cyclophospha- } \\
\text { mide } 600 \mathrm{mg} / \mathrm{m} 2\end{array}$ & HR \pm / HER2 NI & 80 & 16 & 5,6 \\
\hline Conte & 2004 & $\begin{array}{c}\mathrm{D}=\text { epirubicin } 120 \mathrm{mg} / \mathrm{m} 2+\text { paclitaxel } 250 \\
\mathrm{mg} / \mathrm{m} 2 \mathrm{q} 3 \mathrm{w}\end{array}$ & HR \pm / HER2 NI & 94 & 26 & 10,8 \\
\hline Conte & 2004 & $\begin{array}{c}\mathrm{C}=\text { epirubicin } 90 \mathrm{mg} / \mathrm{m} 2+\text { paclitaxel } 200 \\
\mathrm{mg} / \mathrm{m} 2\end{array}$ & HR \pm / HER2 NI & 108 & 20 & 11 \\
\hline Fountzilas & 2004 & $\begin{array}{c}\mathrm{D}=\text { paclitaxel } 175 \mathrm{mg} / \mathrm{m} 2 \mathrm{q} 3 \mathrm{w}+\text { carbopla- } \\
\text { tin AUC of } 6 \mathrm{mg} \mathrm{min} / \mathrm{ml} \mathrm{q3w}\end{array}$ & HR \pm / HER2 \pm & 164 & 27,8 & 10,8 \\
\hline Fountzilas & 2004 & $\begin{array}{c}\mathrm{C}=\text { paclitaxel } 175 \mathrm{mg} / \mathrm{m} 2 \mathrm{q} 3 \mathrm{w}+\text { epirubicin } \\
80 \mathrm{mg} / \mathrm{m} 2 \mathrm{q} 3 \mathrm{w}\end{array}$ & HR \pm / HER2 \pm & 163 & 22,7 & 8,1 \\
\hline Bontenbal & 2005 & $\begin{array}{c}\mathrm{D}=\text { doxorubicin } 50 \mathrm{mg} / \mathrm{m} 2 \mathrm{q} 3 \mathrm{w}+ \\
\text { docetaxel } 75 \mathrm{mg} / \mathrm{m} 2\end{array}$ & HR \pm / HER2 NI & 109 & 22,6 & 8 \\
\hline Bontenbal & 2005 & $\begin{array}{c}\mathrm{C}=\text { fluorouracil } 500 \mathrm{mg} / \mathrm{m} 2 \mathrm{q} 3 \mathrm{w}+\text { doxoru- } \\
\text { bicin } 50 \mathrm{mg} / \mathrm{m} 2 \mathrm{q} 3 \mathrm{w}+\text { cyclophosphamide } \\
500 \mathrm{mg} / \mathrm{m} 2 \mathrm{q} 3 \mathrm{w}\end{array}$ & HR \pm / HER2 NI & 107 & 16,2 & 6,6 \\
\hline Langley & 2005 & $\begin{array}{c}\mathrm{D}=\text { epirubicin } 75 \mathrm{mg} / \mathrm{m} 2 \mathrm{q} 3 \mathrm{w}+\text { paclitaxel } \\
200 \mathrm{mg} / \mathrm{m} 2 \mathrm{q} 3 \mathrm{w}\end{array}$ & $\mathrm{NI}$ & 353 & 13 & 7 \\
\hline Langley & 2005 & $\begin{array}{c}\mathrm{C}=\text { epirubicin } 75 \mathrm{mg} / \mathrm{m} 2 \mathrm{q} 3 \mathrm{w}+\text { cyclophos- } \\
\text { phamide } 600 \mathrm{mg} / \mathrm{m} 2 \mathrm{q} 3 \mathrm{w}\end{array}$ & $\mathrm{NI}$ & 352 & 14 & 7,1 \\
\hline Zielinski & 2005 & $\begin{array}{c}\mathrm{D}=\text { gemcitabine } 1000 \mathrm{mg} / \mathrm{m} 2+\text { epirubicin } 90 \\
\mathrm{mg} / \mathrm{m} 2+\text { Paclitaxel } 175 \mathrm{mg} / \mathrm{m} 2\end{array}$ & HR \pm / HER2 NI & 124 & 29,5 & 9,1 \\
\hline Zielinski & 2005 & $\begin{array}{c}\mathrm{C} \text { = fluorouracil } 500 \mathrm{mg} / \mathrm{m} 2+\text { epirubicin } 90 \\
\mathrm{mg} / \mathrm{m} 2+\text { cyclophosphamide } 500 \mathrm{mg} / \mathrm{m} 2\end{array}$ & HR \pm / HER2 NI & 135 & 24,9 & 9 \\
\hline Albain & 2008 & $\begin{array}{c}\mathrm{D}=\text { gemcitabine } 1250 \mathrm{IV} \mathrm{mg} / \mathrm{m} 2 \mathrm{q} 3 \mathrm{w}+ \\
\text { paclitaxel } 175 \mathrm{mg} / \mathrm{m} 2 \mathrm{q} 3 \mathrm{w}\end{array}$ & HR \pm / HER2 NI & 266 & 18,6 & 5,9 \\
\hline Albain & 2008 & $\mathrm{C}=$ paclitaxel $175 \mathrm{mg} / \mathrm{m} 2 \mathrm{IV}$ q3w & $\mathrm{HR} \pm$ / HER2 NI & 263 & 15,8 & 3,9 \\
\hline Cassier & 2008 & $\begin{aligned} \mathrm{D}= & \text { doxorubicin } 50 \mathrm{mg} / \mathrm{m} 2 \mathrm{q} 3 \mathrm{w}+ \\
& \text { docetaxel } 75 \mathrm{mg} / \mathrm{m} 2 \mathrm{q} 3 \mathrm{w}\end{aligned}$ & HR \pm / HER2 \pm & 107 & 21,4 & 8,7 \\
\hline Cassier & 2008 & $\begin{array}{c}\mathrm{C}=\text { doxorubicin } 50 \mathrm{mg} / \mathrm{m} 2 \mathrm{q} 3 \mathrm{w}+\text { paclitaxel } \\
175 \mathrm{mg} / \mathrm{m} 2 \mathrm{q} 3 \mathrm{w}\end{array}$ & HR \pm / HER2 \pm & 103 & 27,3 & 8 \\
\hline Di Leo & 2008 & $\mathrm{C}=$ paclitaxel $175 \mathrm{mg} / \mathrm{m} 2 \mathrm{IV}$ q3w + placebo & $\mathrm{HR} \pm$ / HER2 \pm & 288 & 20,2 & 5,3 \\
\hline Fountzilas & 2009 & $\begin{array}{c}D=\text { paclitaxel } 175 \mathrm{mg} / \mathrm{m} 2 \mathrm{q} 3 \mathrm{w}+\text { carbopla- } \\
\text { tin AUC of } 6 \text {, in } 500 \mathrm{~mL} \text { q3w }\end{array}$ & HR \pm / HER2 \pm & 136 & 29,9 & 11,5 \\
\hline Fountzilas & 2009 & $\mathrm{C}=$ paclitaxel $80 \mathrm{mg} / \mathrm{m} 2$ weekly & HR \pm / HER2 \pm & 136 & 41 & 11,4 \\
\hline Fountzilas & 2009 & $\begin{array}{c}\mathrm{D}=\text { gemcitabine } 1000 \mathrm{mg} / \mathrm{m} 2 \mathrm{q} 3 \mathrm{w}+ \\
\text { docetaxel } 75 \mathrm{mg} / \mathrm{m} 2 \mathrm{q} 3 \mathrm{w}\end{array}$ & HR \pm / HER2 \pm & 144 & 26,9 & 10,4 \\
\hline Gradishar & 2009 & $\mathrm{C}=$ docetaxel $100 \mathrm{mg} / \mathrm{m} 2 \mathrm{q} 3 \mathrm{w}$ & $\mathrm{NI}$ & 76 & 26,6 & 7,5 \\
\hline Yardley & 2009 & $\mathrm{C}=$ docetaxel $36 \mathrm{mg} / \mathrm{m} 2 \mathrm{IV}$ q4w & $\mathrm{HR} \pm$ / HER2 NI & 52 & 15,4 & 5,5 \\
\hline
\end{tabular}


A despeito das limitações que abordaremos abaixo, a sobrevida global de 15 meses observada nesta análise parece inferior aos resultados da literatura, especialmente quando se avaliam pacientes tratadas depois do advento dos tratamentos alvo dirigidos, momento a partir do qual os resultados melhoraram de forma significativa. De forma análoga, a SLP ou a troca de tratamento para um regime de segunda linha na base de dados acontece num prazo mais curto do que a maior parte das publicações que exploram esta questão. Isto é particularmente importante pois existem evidências que sugerem que uma SLP inferior a 6 meses com a primeira linha de tratamento está associada com menos chances de benefícios com quaisquer tratamentos subsequentes. ${ }^{15}$

Todavia, importantes ressalvas devem ser feitas em relação aos resultados aqui apresentados, uma vez que a SG e a SLP reportadas nesta análise são inferidas a partir de suposições passíveis de importantes vieses, tais como:

- A inferência de progressão da doença com base na troca de tratamento pode superestimar a progressão, uma vez que os pacientes podem, por exemplo, mudar de tratamento por intolerância, informação esta não disponível na base de dados;

- Não há documentação oficial sobre a morte dos pacientes no banco de dados, e o óbito foi estimado a partir do momento em que os pacientes deixam de ter registros adicionais documentados na base de dados para qualquer outro diagnóstico ou procedimento. Isso subestima a SG, porque os pacientes sobrevivem por algumas semanas ou meses em cuidados paliativos após a interrupção do último tratamento e podem não realizar nenhum procedimento ou exame adicional durante este período;

- Os pacientes já podem ter iniciado o tratamento de primeira linha no setor privado e, em seguida, migrar para o sistema público, o que implicaria em SG e SLP mais curtas;

- Os pacientes podem migrar para o ambiente privado após o início do tratamento no ambiente público (portanto, apareceriam na análise com um seguimento mais curto);

- Pode haver preenchimento incorreto dos dados que alimentam a base de dados.

- Porém, em que pesem as limitações dessa análise, a magnitude da diferença entre os 15 meses de SG observados na base de dados do SUS e os 20,8 ou 29 meses reportados na literatura em pacientes diagnosticadas entre 1994 e 2009 traz à tona o questionamento a respeito da qualidade assistencial que estamos oferecendo aos pacientes com câncer de mama no SUS.
Além disso, estima-se que $20 \%$ dos pacientes aqui analisados apresentem tumores HER2-positivos, porém esta informação não se encontra disponível na base de dados. Desde o ano de 2001, tem-se informações sobre ganho de sobrevida e de qualidade de vida com as terapias anti-HER2 ${ }^{16,17,18,19,20}$, tornando-as recomendadas por diretrizes nacionais e internacionais. ${ }^{21,22,23}$ As terapias alvo anti-HER2 atualmente disponíveis no Brasil incluem trastuzumabe $e^{17,24}$, pertuzumabe $20,22,23,24$, lapatinibe ${ }^{25}$ e trastuzumabe entansina ${ }^{19}$, porém nenhum destes tratamentos era disponibilizado pelo SUS no contexto da doença metastática no período avaliado. Assim, a coorte de pacientes avaliada neste estudo recebeu regimes múltiplos de quimioterapia e/ou hormonioterapia, sem nenhuma opção de terapia alvo e é pouco provável que uma percentagem significativa deste grupo de pacientes tenha utilizado tratamentos através de recursos próprios ou por outras vias, porém esta informação também não está disponível. Apenas à título de exemplificação, dados do estudo CLEOPATRA demonstram que pacientes com tumores HER2-positivos avançados que receberam regime baseado em pertuzumabe em primeira linha atingiram SG mediana de 56,5 meses, uma sobrevida também bastante acima da observada nesta coorte. Destaca-se aqui o grande avanço obtido com a publicação da portaria N 29 de 02 de agosto de 2017 que incorpora o trastuzumabe para o tratamento do câncer de mama metastático em primeira linha ${ }^{26}$.

Outra observação digna de nota é o fato de que apenas $5,8 \%$ dos pacientes iniciaram tratamento com hormonioterapia de primeira linha, situação que se contrapõe ao observado na prática clínica internacional e nas instituições dos autores. Todavia, para melhor compreensão deste achado seriam necessárias análises mais específicas e aprofundadas utilizando dados primários. Em estudo publicado pelo LACOG no San Antonio Breast Cancer Symposium de 2016 que avaliou 690 pacientes com câncer de mama avançado em 18 instituições no Brasil, aproximadamente $50 \%$ das pacientes com receptor hormonal positivo utilizavam hormonioterapia em primeira linha .

Apesar das evidentes limitações já anteriormente pontuadas, a informação gerada por esta análise é de extrema relevância para basear discussões sobre a avaliação do padrão atual de tratamento e os desfechos obtidos com os tratamentos atualmente disponíveis em uma coorte brasileira de pacientes tratados pelo SUS, bem como para destacar possíveis áreas de melhoria nos dados coletados e disponibilizados publicamente através do DATASUS. 


\section{CONCLUSÃO}

A sobrevida global e sobrevida livre de progressão inferidas para pacientes com câncer de mama metastático iniciando tratamento de primeira linha no SUS entre janeiro de 2009 e julho de 2010 foi infe- rior aos resultados encontrados por estudos internacionais, o que pode estar em parte relacionado à defasagem do padrão de tratamento disponibilizado para estes pacientes no SUS, em especial no cenário de pacientes HER2 positivo.

\section{REFERÊNCIAS BIBLIOGRÁFICAS}

1. Brasil. Ministério da Saúde. Instituto Nacional de Câncer José Alencar Gomes da Silva (INCA). Estimativa 2016: incidência de câncer no Brasil. Rio de Janeiro: INCA; 2016. 51 p.

2. Cardoso F, Harbeck N, Fallowfield L, Kyriakides S, Senkus E; ESMO Guidelines Working Group. Locally recurrent or metastatic breast cancer: ESMO clinical practice guidelines for diagnosis, treatment and follow-up. Ann Oncol. 2012;23 Suppl 7:vii11-9.

3. Brasil. Ministério da Saúde. Instituto Nacional de Câncer José Alencar Gomes da Silva (INCA). Estimativa 2014: incidência de câncer no Brasil. Rio de Janeiro: INCA; 2014. 124 p.

4. World Health Organization (WHO). Globocan 2012: Breast - Estimated mortality, all ages [Internet]. 2012 [cited 2018 Mar 13]. Available from: http://globocan.iarc.fr/old/summary_table_site-html.asp?selection=3152\&title=Breast\&sex $=2 \&$ type $=1 \&$ window $=1$ \&africa $=1$ \&america $=2 \& a-$ sia=3\&europe $=4 \&$ oceania $=5 \&$ build $=6 \&$ sort $=0 \&$ submit $=\%$ C2\%A0Execute

5. Brasil. Ministério da Saúde. Instituto Nacional de Câncer (INCA). Condutas do INCA - Câncer de mama. Rev Bras Cancerol. 2001;47(1):9-19.

6. Weigelt B, Peterse JL, Van 't Veer LJ. Breast cancer metastasis: markers and models. Nat Rev Cancer. 2005;5(8):591-602.

7. Verma S, Joy AA, Rayson D, McLeod D, Brezden-Masley C, Boileau JF, et al. HER story: the next chapter in HER-2-directed therapy for advanced breast cancer. Oncologist. 2013;18(11):1153-66.

8. Simon SD. Projeto Amazona: Dados preliminares de câncer de mama no Brasil [Internet]. Manaus: GBECAM; 2008 cited 2018 Mar 15]. Available from: www.gbecam.org.br/downloads/Projeto_ Amazona.ppt

9. Sociedade Brasileira de Oncologia Clínica (SBOC). Manual de Condutas 2011. Rev Soc Bras Oncol Clín. 2011; 589 p.

10. Cruz M, Buzaid A, Barrios C, Mayer I. Câncer de Mama [Internet]. Manual de Oncologia Clínica do Brasil. Mama. Doença Metastática; 2016 [cited 2018 Mar 5]. Available from: https://mocbrasil.com/moc-tumores-solidos/cancer-de-mama/ 3-mama-doenca-metastatica/
11. Brasil. Ministério da Saúde. Conselho Nacional de Saúde. Resolução no 510, de 07 de abril de 2016 [Internet]; 2016 [cited 2018 Mar 5]. Available from: http://conselho.saude.gov.br/resolucoes/2016/Reso510.pdf

12. Andre $F$, Slimane $K$, Bachelot $T$, Dunant $A$, Namer M, Barrelier A, et al. Breast cancer with synchronous metastases: trends in survival during a 14year period. J Clin Oncol. 2004;22(16):3302-8.

13. Muss HB, Case LD, Richards F 2nd, White DR, Cooper MR, Cruz JM, et al. Interrupted versus Continuous Chemotherapy in Patients with Metastatic Breast Cancer. The Piedmont Oncology Association. N Engl J Med. 1991;325(19):1342-8.

14. Adunlin G, Cyrus JW, Dranitsaris G. Correlation between progression-free survival and overall survival in metastatic breast cancer patients receiving anthracyclines, taxanes, or targeted therapies: a trial-level meta-analysis. Breast Cancer Res Treat. 2015;154(3):591-608.

15. Bonotto M, Gerratana L, Iacono D, Minisini AM, Rihawi K, Fasola G, et al. Treatment of Metastatic Breast Cancer in a Real-World Scenario: Is Progression-Free Survival With First Line Predictive of Benefit From Second and Later Lines? Oncologist. 2015;20(7):719-24.

16. Slamon D, Eiermann W, Robert N, Pienkowski T, Martin M, Press M, et al.; Breast Cancer International Research Group. Adjuvant trastuzumab in HER2-positive breast cancer. N Eng J Med. 2011;365(14):1273-83.

17. Swain SM, Baselga J, Kim SB, Ro J, Semiglazov V, Campone M, et al.; CLEOPATRA Study Group. Pertuzumab, trastuzumab, and docetaxel in HER2-positive metastatic breast cancer. N Engl J Med. 2015;372(8):724-34.

18. Verma S, Miles D, Gianni L, Krop IE, Welslau M, Baselga J, et al.; EMILIA Study Group. Trastuzumab emtansine for HER2-positive advanced breast cancer. New Engl J Med. 2012;367(19):1783-91.

19. Baselga J, Cortés J, Kim S, Im S, Hegg R, Im YH, et al.; CLEOPATRA Study Group. Pertuzumab plus trastuzumab plus docetaxel for metastatic breast cancer. N Eng J Med. 2012;366(2):109-19.

20. Geyer CE, Forster J, Lindquist D, Chan S, Romieu CG, Pienkowski T, et al. Lapatinib plus capecitabine for HER2-positive advanced breast cancer. N Engl J Med. 2006;355(26):2733-43. 
21. NCCN Clinical Practice Guidelines in Oncology Breast Cancer. v3.2015. [Internet]. [cited 2015 Dec 4]. Available from: http://www.nccn.org/professionals/physician_gls/pdf/breast.pdf

22. Cardoso F, Costa A, Norton L, Senkus E, Aapro M, André $\mathrm{F}$, et al. ESO-ESMO 2nd international consensus guidelines for advanced breast cancer (ABC2). Ann Oncol. 2014;25(10):1871-88.

23. Giordano SH, Temin S, Kirshner JJ, Chandarlapaty S, Crews JR, Davidson NE, et al.; American Society of Clinical Oncology. Systemic therapy for patients with advanced human epidermal growth factor receptor 2-positive breast cancer: American Society of Clinical Oncology clinical practice guideline. J Clin Oncol. 2014;32(19):2078-99.
24. Jiang $H$, Rugo HS. Human epidermal growth factor receptor 2 positive (HER2+) metastatic breast cancer: how the latest results are improving therapeutic options. Ther Adv Med Oncol. 2015;7(6):321-39.

25. Geyer CE, Forster J, Lindquist D, Chan S, Romieu CG, Pienkowski T, et al. Lapatinib plus capecitabine for HER2-positive advanced breast cancer. N Engl J Med. 2006;355(26):2733-43.

26. Barrios $\mathrm{CH}$, Uema D, Cronenberger E, Lima $\mathrm{V}$, Bines J, de Santsana RO, et al. Abstract P6-16-04: Real World data and patterns of care of metastatic breast cancer (MBC) in Brazil: First results of LACOG 0312 retrospective study. Cancer Res 2017;77(Suppl. 4):Abstract P6-16-04. 